\title{
Integrated Health Log: Share Multimedia Patient Data
}

\author{
Rogier Brussee, \\ Paul Porskamp \\ Telematica Instituut \\ P.O. Box 589, \\ 7500 AN Enschede, \\ The Netherlands \\ Rogier.Brussee@telin.nl, \\ Paul.Porskamp@telin.nl
}

\author{
Leon van den Oord, \\ Erik Rongen \\ IBM Dynatos, \\ David Ricardostraat 2-4, \\ 1066 JS Amsterdam, \\ The Netherlands \\ Leon.vandenOord@nl.ibm. \\ com, \\ Erik@nl.ibm.com
}

\author{
Hans Bloo, \\ Victorien Erren, \\ Leendert Schaake \\ Roessingh Research and \\ Development, \\ P.O. Box 310 \\ 7500 AH Enschede, \\ The Netherlands \\ H.Bloo@rrd.nl, \\ V.Erren@rrd.nl \\ L.Schaake@rrd.nl
}

\begin{abstract}
The Integrated Health Log demonstrator shows how multimedia can be used in collaborative settings in healthcare. Patient data can be shared annotated discussed and processed by medics involved with a patient. Special emphasis is put on protecting the privacy of patients and allowing medics to keep responsibility for their patient data. In particular, grid technology is used to decouple services and service providers so that services can be executed under the control of the data owner. The system will be piloted in a gait analysis laboratory and in a network of physiotherapists involved in treating children with movement disorders.
\end{abstract}

\section{Introduction}

Patient records are an integral part of diagnosis and treatment in healthcare and documentation is an established part of its workflow. Records are of particular importance when several professionals are involved in diagnosing and treating a patient (multidisciplinary integrated healthcare) to avoid clinical errors and as a basis for effective communication. Increasingly, patient data involves multimedia data. For example physiotherapists regularly use patient data that contains video, images and biosensor data.

In the MultimediaN Integrated Health Log (IHL) project from IBM, Roessingh Research and Development and the Telematica Instituut, we are developing an open source infrastructure to manage, share and use multi media patient data. Special emphasis is given to a version for physiotherapists called Physiolog. In the laboratory, IHL gives an improved workflow, synchronized bio-data and video or image analysis support for gait analysis. Networks of collaborating therapists can share multimedia content for diagnostic or training purposes.

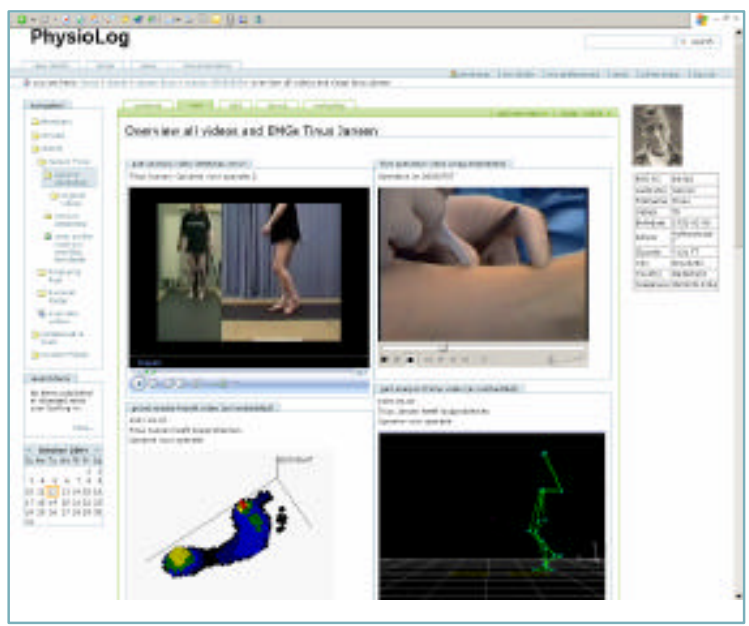

Figure 1: Patient data with gait video, operation video visualised pressure plate data and VICOM stream. 
The project stresses the importance of controlling access to patient data by people and services. While IHL encourages both sharing of patient data with other professionals and operating on content and data, medics are required by law to protect the privacy of their patients. Thus we are building mechanisms that make access a matter of trust, choice and policy rather than the result of "coincidental" technical circumstances. In particular we use grid technology for building external services that are instantiated at and communicating with sites trusted by the data owner only.

\section{Related Work}

The work in IHL builds on previous work in the Telefysi project [3]. There has been extensive work on systems for multimedia patient in clinical settings (e.g. [1],[2],[7],[13]). Work on models for dealing with sensitive access patient data can be found in [6],[5] and [10] where the latter focuses more on organizational issues.

\section{The Integrated Health Log application}

The Integrated Health Log application is build around a content management system (CMS) which interfaces with common collaborative groupware tools.

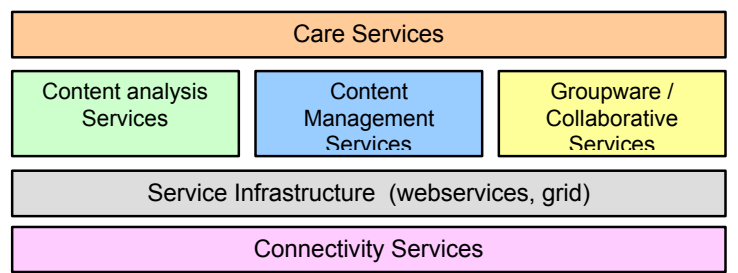

Figure 2: Integrated Health Log top level architecture

Using standards based off the shelf open source or commercial components makes the system easy to use and install, and means that there is a large body of available, and ever growing, "mundane" functionality. In particular, the content management layer allows the definition of various roles such as therapist, collaborator, patient, service provider or administrator that can be used to define a workflow and to finely control access to content. The collaborative layer allows for discussing content between therapists and, if desired, communication between therapist and patient.

On top of the CMS there is a layer of care services responsible for interacting with therapists and dealing with special medical data. These care services are domain specific and range from forms for an examination workflow, to the services that interpret raw sensor data. Providing different such services is what distinguishes various incarnations of IHL such as Physiolog, although of course some care services can be reused in other specializations.

In addition to such care services there is a layer to interface the CMS with external (grid) services, for example to analyze multi media data. External services are instantiated wherever the patient data owner trusts the execution and the required technical environment is available. Given those hard requirements we will choose the computer system that will give results soonest and/or is the cheapest. Currently we have only simple video transformation and analysis methods available but we plan to use more sophisticated image analysis services to help with physiotherapists gait analysis.

The Plone/Zope [9] based open source Integrated Health Log software has been attracting interest from other fields such as sports and psychotherapy and the project is branching out.

\section{A usage scenario}

Sybil is a physiotherapist treating children with a motion disorder. She is currently involved with a nine year old patient Jan, who has both physical and mental disorders. Jan's motor problems have resulted in several bone injuries which she would like to take into account. Jan is also being treated by an orthopedist and a neurologist. Fortunately, Jan's parents are willing to sign permission for passing the orthopedists and neurologists data to her. Once the signatures are in, she uses the data to make a treatment plan which she discusses with her peers. The discussion results in some hints from a highly appreciated colleague which she takes into account for the final treatment plan.

After a number of treatments Jan has another accident and breaks his kneecap. Once the plaster comes off the hospital orthopedist Heracles requests a detailed gait analysis at the hospital's movement laboratory where a video is made and EMG sensor data is collected. Victoria, the hospital's physiotherapist routinely runs the video and the sensor data through the national revalidation center's analysis software that can run on the large hospital server. She writes a report and points out that Jan overstretches his left knee by more than 10 degrees. At Heracles request she makes the report and her other data available to Sybil. 
Sybil is involved with a clinical study on knee injuries. She asks and gets permission from the parents to use Jan's anonymised data for this study. In the web fate Jan becomes patient number 713 .

\section{Pilots}

Physiolog will be piloted in the field in a clinical movement analysis laboratory at Roessingh Research and development, and in a network of therapists, responsible for the treatment of child movement disorders in Twente, the Netherlands.

The laboratory pilot has recently been deployed, and has already proved to be of considerable benefit for the workflow of the examination. Patient data is gathered life during a patient's gait analysis. Therapists fill in the results of physical examinations and clinical observations on a special purpose form, modeled after existing paper forms.

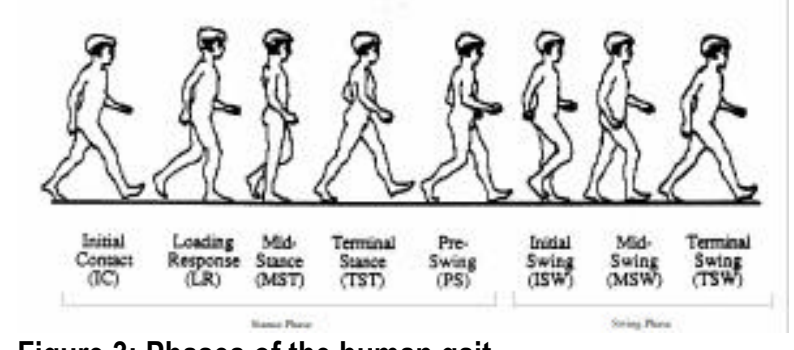

Figure 3: Phases of the human gait

Videos of the patients gait under controlled circumstances are made for subsequent analysis by the therapist and as a basis for diagnosis. Moreover for some patients sensor data is collected such as EMG (ElectroMyoGraphy) data measuring the electrical activity of motor nerves in muscles, foot pressure data, or VICON streams that determine the 3 dimensional position of fixed parts of the body during the gait.

Both video and sensor data are stored in the system and are associated to the patient and a session. The final diagnosis is usually send to a requesting treating doctor. In addition to current practice where the diagnosis is simply described in structured natural language, the Physiolog system allows other medics to access all data, including multimedia and sensor data. This means that the diagnosing therapist can include links to specific parts of videos or point to sensor data. In a later stage we hope to help objectify a diagnosis by using image analysis on the videos of the gait, for example to determine the precise moments of touchdown of the foot or the bending angle of the knee.

Patient data is stored per session and patient as befits the workflow in the laboratory. However results are made searchable for clinical studies on various clinical characteristics. Such different views on data reflect that data is used in different ways by different people in different roles, and the system supports controlling access based on a user's role.

In the network pilot that is currently being set up, 20 therapists specializing in child therapy will gather casuistic and share experiences building on previous work in the Telefysi project [3]. Since some disorders are rare, sharing special cases is an important part of continuous therapist training. Moreover, even in more common cases it is considered good professional conduct to get second opinions. The Physiolog system will be used to manage and store patient cases, and will benefit directly from the work done for the laboratory case. The network case is also a good example of the use of patient data by several parties crossing organizational boundaries.

\section{Trust model and virtual administrative domains.}

Different actors in healthcare play a different role and have different spheres of influence. This is reflected in the organizations and sub-organizations that actors are affiliated to. Such an organization often supports its workflow with an ICT system with a uniform administrative domain. Typically, within the organization, people trust each other more than people outside of the organization. Therefore the administrative domain gives members of the organization special privileges and access rights. However in practice this means that such administrative domains are centered on treating medics which makes it more difficult to provide a patient centered view. Such a view is useful if the patient is treated by several medics that may not share the same administrative domain and helps in collaborations.

The physiology trust model assumes that a virtual administrative domain on top of the existing administrative domains is created as a software construct. Within this virtual domain access rights to patient data can be managed in a uniform way. We stress that the virtual administrative domains should reflect real trust and support real cooperation between real people or organizations that have made some real 
agreements. Its intention is to make it (more) practicable to work with distributed patient data and loosely coupled independent systems under local control rather than a centralized server. This fits well with current architectural thinking on linking electronic patient data (see [8],[12]). The approach is heavily influenced by ideas in the grid community on virtual organizations [4]. Indeed we use the same mechanisms and need virtual administrative domains that include the providers of resources such as computation, data and services. However rather than emphasizing large scale public utility deployments, to use available computing resources and controlling access to hardware, our emphasis is on small deployments with recognizable responsibilities to use available external software services and controlling access to patient data.

The access control mechanisms of the CMS are used as a model and an available mechanism that is extended to access control service instantiations. This is achieved by translating access restrictions into SAML (security assertion markup language) messages [11] that are handed to the grid security infrastructure. Access restrictions are upheld locally under the responsibility of the owners of data of resources, according to guiding principle that whoever is responsible for data or resources gets a final say over who gets access. However, if access restrictions are made public, resource and discovery frameworks can take these restrictions into account for efficiency reasons.

\section{Conclusion.}

We have presented a system for managing multimedia patient data. Special emphasis is given to respecting the privacy of patients while still allowing advanced operation on patient data. We propose a scheme for dealing with distributed sensitive data, virtual administrative domains, to support this. The system is and will be further piloted by physiotherapists in the Netherlands.

\section{References}

[1] Dayhoff, R., Kuzmak, P.M. Kirin, G. Frank, S. "Providing a Complete Online Multimedia Patient Record" Proc. AMIA Symp. 1999. Available at http:/www.amia.org/pubs/symposia/D005623.PDF [2] Dayhoff, R.E.., Kuzmak, P.M. Frank, S. "The multimedia electronic patient record: current issues" proc. AMIA annual symp. 2003; p1063

[3] Engbers, L., Bloo, H., Kleissen, J., Spoelstra, M., Vollenbroek-Hutten, M. "Development of a teleconsultation system for communication between Physiotherapists concerning children with complex movement and postural disorders." Journal of Telemedicine and Telecare. $2003 \mathrm{vol}$. 9 no 6 pp 339-343. Available at http:/www.ingentaconnect.com/search/expand?pub=infobike: //rsm/jtt/2003/00000009/00000006/art00005

[4] Foster, I. Kesselman, C., Tuecke, S. "The anatomy of the Grid.”, Int. J. Supercomputer App. 15(3) 2001. Available at http://www-

unix.globus.org/alliance/publications/papers/anatomy.pdf [5] Georgiadis C.K., Mavridis, I., Pangalos, G., Thomas, R. "Flexible Team Based Access Control Using Contexts." SACMAT 2001 (ACM Workshop on Role based Access Control, 2001). Available at

http://portal.acm.org/citation.cfm?coll=Guide\&dl=Guide\&id $=373259$ and

http://infolab.gen.auth.gr/Phd/mavridis/SACMAT2001.pdf

[6] Louwerse, K. "Access control management in practical settings." Stud Health Technol. Inform. 2004; 103 pp 424427

[7] Lowe, H.J. Multimedia electronic medical record systems. Acad. Med. 1999 Feb, 74(2), pp.146-152. Available at

http://www.ncbi.nlm.nih.gov/entrez/query.fcgi?cmd=Retriev e \&db=PubMed\&list uids $=10065056 \&$ dopt $=$ Citation [8] Nictiz. "Architectuurontwerp basisinfrastructuur" Nictiz 2004. Available at

http://www.nictiz.nl/kr nictiz/uploaddb/downl object.asp?at oom $=3175 \& \mathrm{VolgNr}=1$

[9] Plone site : Available at http://plone.org

[10] Rind M. et al. "Maintaining the Confidentiality of Medical Records Shared over the Internet and the World Wide Web." Ann of Int. Medicine July 1997, 127(2) pp. 138141. Available at www.annals.org/content/full/127/2/138

[11] OASIS security services site : http://www.oasisopen.org/committees/tc home.php?wg_abbrev=security [12] Shirky, C. et al. "Linking health care information: proposed methods for improving care and protecting privacy." Connecting for Health, working group on accurately linking information for healthcare quality and safety. Available at

http://www.connectingforhealth.org/assets/reports/linking_re port 2 2005.pdf

[13] Wang, SS., Staren J. "A web based, secure, lightweight clinical multimedia data capture and siplay system" Proc. AMIA symp. 2000, pp 898-902. 